\title{
The reciprocal antagonistic interplay between HIV-1 Tat and SIN3A specifies the epigenetic switch between de-repression versus maintenance of HIV-1 gene silencing
}

\author{
Anurag Kulkarni ${ }^{1 *}$, Estelle Villemaine ${ }^{1}$, Ann Marie McCartin ${ }^{1}$, Elena Woods ${ }^{1}$, Celine Marban $^{2}$, Virginie Gautier ${ }^{1}$
}

From Frontiers of Retrovirology: Complex retroviruses, retroelements and their hosts

Cambridge, UK. 16-18 September 2013

\section{Background}

Post-integration latency is a critical barrier to achieving a complete cure for HIV. Epigenetic micro-environment alterations at the integrated HIV-1 LTR, coupled with modulations of the HIV-1 Tat feedback circuit, influence the onset and maintenance of latency. In this context, we have previously identified the epigenetic core pressor SIN3/HDAC complex as part of the in-vitro HIV-1 Tat nuclear interactome. Here we delineate the dynamics of their antagonistic functional relationship in the control of HIV-1 latency.

\section{Methods}

Well-studied T-cellular models of HIV-1 latency viz. J-LAT A1 \& A72, with latent integrated LTR-Tat-IRESGFP-LTR and LTR-GFP-LTR cassettes respectively, and $\mathrm{ACH}-2$, with latent full-length integrated HIV-1 provirus in their genome, were employed. We used ChIPqPCR to study protein occupancy at the HIV-1 LTR; and RT-qPCR and p24 ELISA/GFP FACS to concurrently monitor the reactivation of HIV-1 gene expression. SIN3A knock-down was performed with shRNA targeting sin3a or non-target control using Amaxa nucleofection, and was validated by WB and RT-PCR analysis. Immunofluorescence microscopy was performed in Hela cells co-transfected with Tat and Sin3a.

\section{Results}

The latent HIV-1 LTR displays a constitutive enrichment of the SIN3/HDAC complex.

'UCD-Centre for Research in Infectious Diseases (CRID), School of Medicine and Medical Science, University College Dublin, Dublin, Ireland

Full list of author information is available at the end of the article
ChIP-qPCR revealed that the SIN3/HDAC key components SIN3A, SAP18, Sap3O and HDAC-1 were enriched in ACH-2, J-LAT A1 and A72 cells. Importantly, a key event in HIV-1 reactivation from latency is the displacement of the SIN3/HDAC complex from the HIV-1 LTR, as TNF $\alpha$ (NF-KB activator), 5-AZA (DNA methylation inhibitor) or SAHA (HDAC inhibitor) treatments disrupted SIN3/HDAC association with the HIV-1 LTR in latently infected cells.

SIN3A is critical for the maintenance of HIV-1 post-integration latency. SIN3A depletion in J-LAT A1 and A72 resulted in HIV-1 reactivation from latency, which was associated with the depletion of SIN3A and concurrent recruitment of POLII and/or HIV-1 Tat, at the HIV-1 LTR. Next we combined SIN3A knock-down with various reactivation treatments and observed that SIN3A depletion potentiated proviral reactivation by $\mathrm{TNF} \alpha, \mathrm{PMA} /$ ionomycin (Protein Kinase $\mathrm{C}$ activator), 5-AZA and SAHA in J-LAT A1 cells. Thus when associated with the HIV-1 LTR, SIN3A participates in the silencing of the provirus.

\section{Authors' details \\ 'UCD-Centre for Research in Infectious Diseases (CRID), School of Medicine and Medical Science, University College Dublin, Dublin, Ireland. ${ }^{2}$ INSERM U1121, Faculté de Chirurgie Dentaire, Université de Strasbourg, Strasbourg, France.}

Published: 19 September 2013

doi:10.1186/1742-4690-10-S1-012

Cite this article as: Kulkarni et al:: The reciprocal antagonistic interplay between HIV-1 Tat and SIN3A specifies the epigenetic switch between de-repression versus maintenance of HIV-1 gene silencing. Retrovirology 2013 10(Suppl 1):012. 